\title{
Pre-hospital predictors of an adverse outcome among patients with dyspnoea as the main symptom assessed by pre-hospital emergency nurses - a retrospective observational study
}

Wivica Kauppi ${ }^{1,2^{*}}$ (D) Johan Herlitz ${ }^{1,2}$, Thomas Karlsson ${ }^{3}$, Carl Magnusson ${ }^{1,4}$, Lina Palmér ${ }^{2}$ and Christer Axelsson ${ }^{1,2}$

\begin{abstract}
Background: Dyspnoea is one of the most common reasons for patients contacting emergency medical services (EMS). Pre-hospital Emergency Nurses (PENs) are independently responsible for advanced care and to meet these patients individual needs. Patients with dyspnoea constitute a complex group, with multiple different final diagnoses and with a high risk of death. This study aimed to describe on-scene factors associated with an increased risk of a time-sensitive final diagnosis and the risk of death.

Methods: A retrospective observational study including patients aged $\geq 16$ years, presenting mainly with dyspnoea was conducted. Patients were identified thorough an EMS database, and were assessed by PENs in the southwestern part of Sweden during January to December 2017. Of 7260 missions (9\% of all primary missions), 6354 were included. Among those, 4587 patients were randomly selected in conjunction with adjusting for unique patients with single occasions. Data were manually collected through both EMS- and hospital records and final diagnoses were determined through the final diagnoses verified in hospital records. Analysis was performed using multiple logistic regression and multiple imputations.

Results: Among all unique patients with dyspnoea as the main symptom, 13\% had a time-sensitive final diagnosis. The three most frequent final time-sensitive diagnoses were cardiac diseases (4.1\% of all diagnoses), infectious/ inflammatory diseases (2.6\%), and vascular diseases (2.4\%). A history of hypertension, renal disease, symptoms of pain, abnormal respiratory rate, impaired consciousness, a pathologic ECG and a short delay until call for EMS were associated with an increased risk of a time-sensitive final diagnosis. Among patients with time-sensitive diagnoses, approximately $27 \%$ died within 30 days. Increasing age, a history of renal disease, cancer, low systolic blood pressures, impaired consciousness and abnormal body temperature were associated with an increased risk of death. (Continued on next page)
\end{abstract}

\footnotetext{
* Correspondence: wivica.kauppi@hb.se

${ }^{1}$ PreHospen- Centre for Pre-hospital Research, Faculty of Caring, Work Life and Social Welfare, University of Borås, SE-501 90 Borås, Sweden

${ }^{2}$ Faculty of Caring Science, Work Life and Social Welfare, University of Borås, Borås, Sweden

Full list of author information is available at the end of the article
}

(c) The Author(s). 2020 Open Access This article is licensed under a Creative Commons Attribution 4.0 International License, which permits use, sharing, adaptation, distribution and reproduction in any medium or format, as long as you give appropriate credit to the original author(s) and the source, provide a link to the Creative Commons licence, and indicate if changes were made. The images or other third party material in this article are included in the article's Creative Commons licence, unless indicated otherwise in a credit line to the material. If material is not included in the article's Creative Commons licence and your intended use is not permitted by statutory regulation or exceeds the permitted use, you will need to obtain permission directly from the copyright holder. To view a copy of this licence, visit http://creativecommons.org/licenses/by/4.0/ The Creative Commons Public Domain Dedication waiver (http://creativecommons.org/publicdomain/zero/1.0/) applies to the data made available in this article, unless otherwise stated in a credit line to the data. 
(Continued from previous page)

Conclusions: Among patients with dyspnoea as the main symptom, age, previous medical history, deviating vital signs, ECG pattern, symptoms of pain, and a short delay until call for EMS are important factors to consider in the prehospital assessment of the combined risk of either having a time-sensitive diagnosis or death.

Keywords: Dyspnoea, Epidemiology, Adverse outcome, Time-sensitive diagnosis, Ambulance, Emergency medical service, Pre-hospital emergency nurse

\section{Background}

Dyspnoea, also known as shortness of breath or breathlessness, is one of the most common reasons patients contact EMS. Patients with dyspnoea constitute a heterogeneous group since comorbidity is common. Dyspnoea is associated with a high risk of death and is caused by several medical conditions that include physiological, pathological, psychological conditions, or social reasons $[1,2]$. In Sweden, PENs have an important role in the assessment and triage, as well as in the care of patients with dyspnoea on the scene. This assessment challenges the PENs ability to assess the patient properly [3], which requires a comprehensive understanding of the pathophysiology, as well as the ability to meet the patients existential needs since anxiety is common in connection with dyspnoea [4]. Few epidemiological studies have described patients with dyspnoea in pre-hospital settings [5-7]. A recent study by Kauppi et al. [8] described the epidemiology and outcomes of patients with dyspnoea as the main symptom from a larger perspective. In the current study among patients whose main symptom was dyspnoea, we aimed to describe a) timesensitive conditions in further detail and b) the factors that already on scene were associated with the risk of either having a time-sensitive final diagnosis or death within 30 days when receiving care from PENs. The two primary endpoints were thus: 1 . A time-sensitive condition according to the final diagnosis, and 2. Death within 30 days.

\section{Methods}

The results in this study are based on further analysis from the same study population as recently described by Kauppi et al. [8].

\section{Study design}

This is an exploratory observational study with a retrospective manual assessment of EMS and hospital records by one single person. The study included all patients who dialled 112 in Sweden between January and December 2017 with dyspnoea as the main symptom. The call was followed by an ambulance being dispatched to the scene, and the provision of assessment and care by PENs.

\section{Study settings}

The study included two EMS organisations in the southwestern part of Sweden with a catchment population of 962,000 inhabitants and a catchment area of approximately $7400 \mathrm{~km}^{2}$ including both urban, suburban and rural areas. In all, the EMS organisations had 123,614 missions with a priority level of 1 to 3 . Among them, 87, 611 missions where involved in an initial patient assessment that was defined as a primary mission (Fig. 1). In Sweden, all ambulances are since 2005 at a minimum staffed with two health care providers, of which one is a registered nurse [9]. A major part of the nurses have fulfilled a three-year nursing course as well as a one-year master's course that are focusing on pre-hospital emergency care and are given the professional title 'PEN'. The PENs are responsible for care including assessment and treatment according to local and national guidelines.

\section{Patients}

Patients were included by missions through an EMSrecord database and a hospital record system. The EMS recorded database includes the triage classification system (RETTS-A) and the hospital record system contains the International Classification of Diseases (ICD-10) codes, which includes 22 chapters (I-XXII). Electrocardiogram (ECG) interpretations were primarily collected from pre-hospital records, alternatively from Emergency Department (ED) notes, and if not available, interpreted by the first author (WK). ECG abnormalities that were looked for included atrial flutter, atrial fibrillation, STsegment and T-wave deviation, left bundle branch block, ventricular tachycardia, ectopic atrial rhythm or tachycardia, and AV-block. Of all vital signs (VS), the first (on arrival at the patient's side) and last (before admission to ED) assessments were used.

Inclusion criteria were a primary mission where the dispatch centre had been contacted and was given a priority level of $1-3$, and where the mission was classified on scene as dyspnoea as the main symptom, which consists of the Emergency Symptoms and Signs (ESS) code number 04 . Exclusion criteria included age $<16$ years, no personal identity number, an incorrect ESS triage code, transportation to a hospital outside the catchment areas, limited information, EMS records that appeared more 


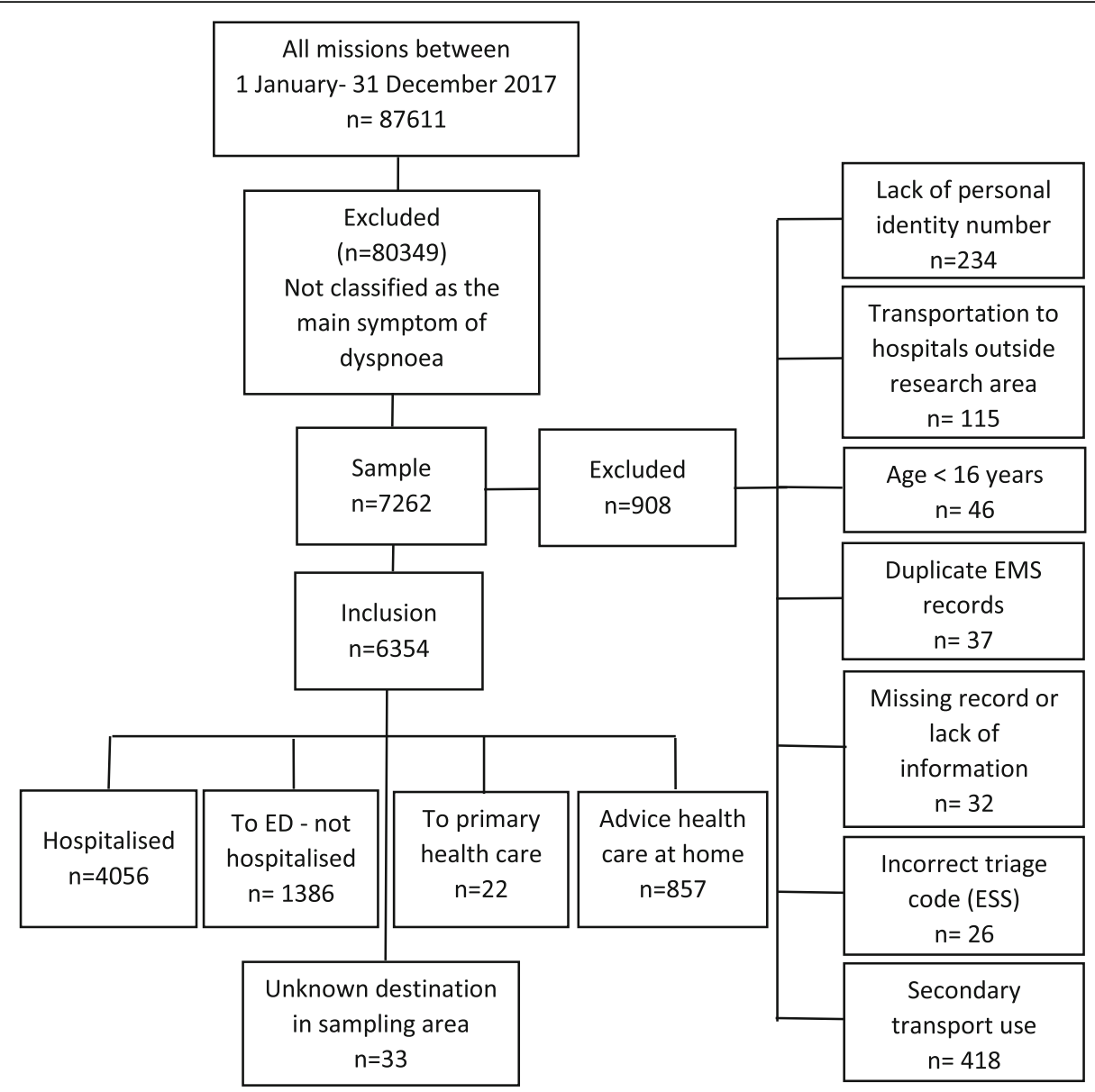

Fig. 1 Flow chart of all studied patients, assessed as the main symptom of dyspnoea

than once and the use of a secondary transport. A total of 7260 patients were identified (9\% of all missions) of which 908 patients were excluded. Thus, altogether 6354 patients took part in the final analyses, some presenting on multiple occasions (Fig. 1). Among these 6354 patients were 4587 randomly selected adjusting for unique patients with single occasions (Fig. 2).

\section{Triage system}

The triage classification system Rapid Emergency Triage and Treatment System for adults (RETTS- A) is used by the PENs to assess and prioritise patients degree of prehospital care required [10]. This system is based on VS and ESS, which both (independently of each other) leads to a recommendation of the priority level. The highest level of either the ESS or VS determines the final triage level [see Additional file 1: Red and orange VS and ESS code 04, dyspnoea]. The priority level is expressed in five different colours (red, orange, yellow, green, and blue), which refers to time from the assessment until time when patients require to be assessed by a physician. Red and orange level indicate the highest priority (life- threatening vs. potentially life-threatening), whereas the remaining colours represent situations when there is no medical risk for patients if the waiting time is prolonged before physician assessment. In the pre-hospital triage, the level blue is not used.

\section{Time-sensitive diagnosis}

We defined a time-sensitive diagnosis as a diagnosis when urgent care and treatment are vital to limit organ damage and to avoid severe complications and early death (e.g. pulmonary oedema, acute myocardial infarction and sepsis) $[3,11]$. This definition has been previously described [3], but some additional diagnoses (e.g. acute respiratory failure and acidosis) that fulfilled the criteria for the above definition were added in the analyses of a recent cohort study by Kauppi et al. [8] and have therefore been included.

\section{Statistical analysis}

Multiple logistic regression was used for calculation of age-adjusted $p$-values and odds ratios with corresponding confidence intervals regarding time-sensitive diagnosis 


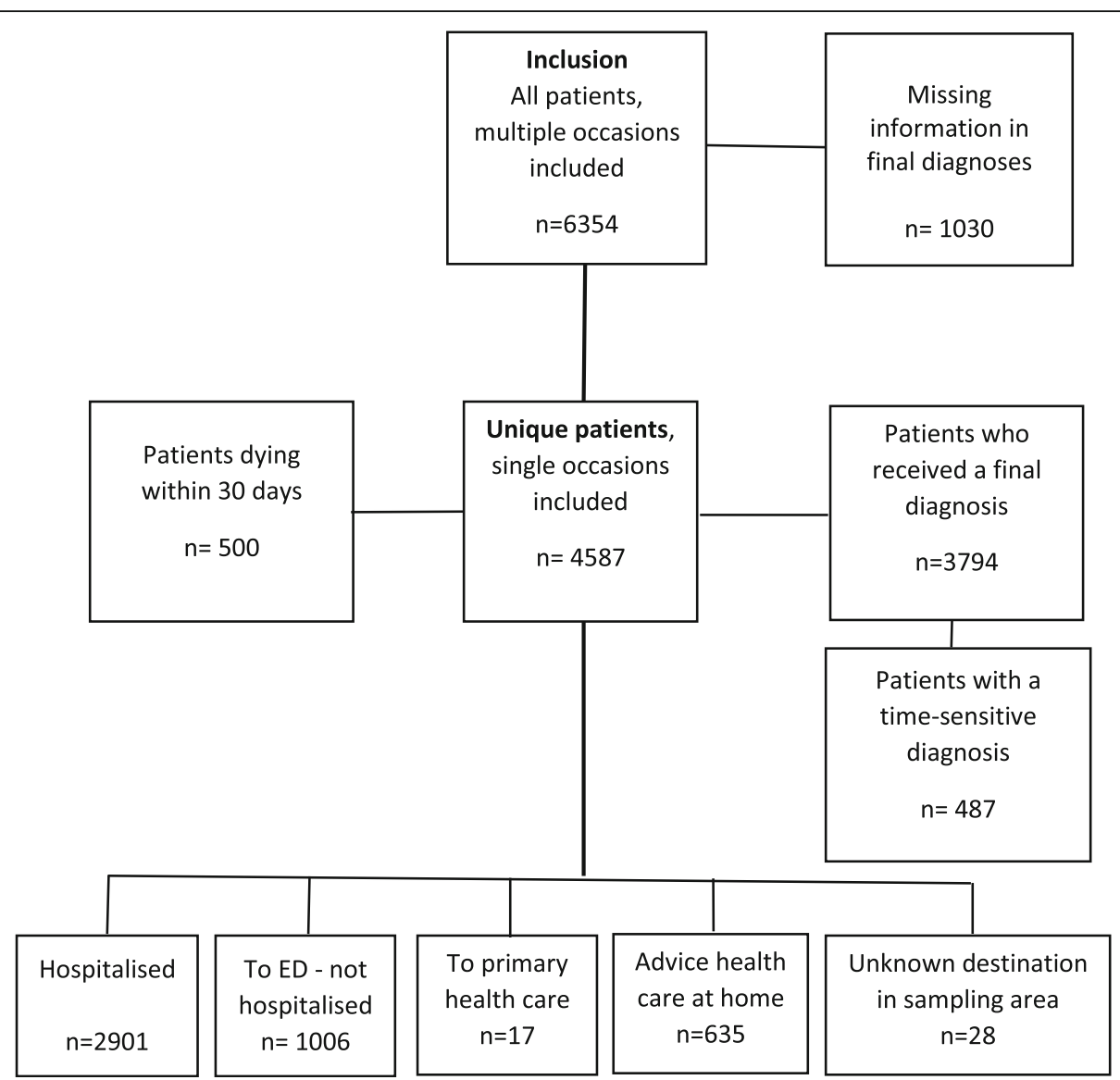

Fig. 2 Flow chart of the included unique patients, single occasions randomly selected, assessed as the main symptom of dyspnoea

and 30-day mortality, respectively. Available data was used for these calculations.

To identify factors independently associated with the two outcomes, all variables with an age-adjusted $p<0.20$ in Tables 2 and 4, respectively, were tested for inclusion in a final multiple logistic regression model. No significant collinearity was found between the variables by association measures, as well as by inspection of the variance inflation factor, condition index, and eigenvector proportions in a multiple linear regression model including all the candidate variables. Due to the amount of missing data for several of the variables, multiple imputations were applied. For these analyses, missing data were assumed to be missing at random (MAR) and 50 imputed datasets were generated with the Markov Chain Monte Carlo (MCMC) method using the expectation-maximization (EM) algorithm. Rubin's rules were used for pooling the results from the imputed datasets. To identify variables independently associated with outcome, we started with a model including all the variables as identified above. Multiple logistic regression was performed in each of the 50 imputed datasets, and the variable with the highest $p$-value in the pooled result was excluded from the model. A new regression analysis was then performed in each imputed dataset and of the remaining variables, the one with the highest $\mathrm{p}$-value in the pooled result was excluded. This procedure was repeated until all remaining variables yielded a p-value below 0.01 in the pooled result. This was performed separately for the timesensitive diagnosis and 30-day mortality outcomes. Twosided tests were used and $p$-values below 0.01 were considered statistically significant. All analyses were performed using SAS for Windows version 9.4.

\section{Results}

In all, 6354 EMS missions were included (Fig. 1). Among them, there were 4587 unique patients with dyspnoea as the main symptom, of which 3794 had a final diagnosis. Time-sensitive final diagnoses were reported in 487 cases, which was approximately $13 \%$ of all unique patients with a final diagnosis (Fig. 2), and among them approximately $27 \%$ died within 30 days.

\section{Occurrence of time-sensitive diagnosis among all missions with a final diagnosis}

The most frequent final time-sensitive diagnoses among all missions were cardiac diseases ( $4.1 \%$ of all diagnoses) 
followed in order of frequency by infectious/inflammatory diseases $(2.6 \%)$, vascular diseases $(2.4 \%)$, respiratory diseases $(1.5 \%)$, and neurological diseases (0.3\%).

The cardiac and infectious time-sensitive diagnoses were more common in males and the elderly whereas the vascular and the respiratory time-sensitive diagnoses were most frequent in females and younger patients. Of all missions with a final diagnosis $(n=5324$ missions), $11.5 \%$ had a time-sensitive diagnosis (Table 1 ).

\section{Age-adjusted relationships between time-sensitive} diagnosis and sex, previous history, time intervals, and clinical observations on arrival of PENs, as well as age itself, among unique patients with a final diagnosis

Time-sensitive diagnoses among unique patients with a final diagnosis were reported in approximately $13 \%$ of all cases. Among them, the most common clinical deviations from normal were an abnormal respiratory rate (72.3\%), a low oxygen saturation (59.5\%), and a pathological ECG (52.6\%). The following were significantly associated with an increased risk of a time-sensitive diagnosis: a history of hypertension, diabetes and renal disease, recent or ongoing syncope, abnormalities in any of the six VS, and a pathological ECG.

The following were significantly associated with a decreased risk: a history of dyspnoea, atrial fibrillation, and pulmonary disease, increasing interval from onset of symptoms until the call for EMS and time from the call for EMS to PENs arrival (Table 2).

Multivariable analysis of factors independently associated with a time-sensitive diagnosis using multiple imputations (3794 patients, 487 with a time-sensitive diagnosis)

The following were significantly independently associated with an increased risk of a time-sensitive diagnosis among unique patients: a history of hypertension and renal disease, symptoms of pain, an abnormal respiratory rate, a low oxygen saturation, an abnormal heart rate, a decreased level of consciousness and a pathologic ECG.

Table 1 Occurrence of time-sensitive diagnosis among all missions with a final diagnosis

\begin{tabular}{|c|c|c|c|c|c|}
\hline & $\begin{array}{l}\text { All patients } \\
(n=5324)^{\mathrm{a}}\end{array}$ & $\begin{array}{l}\text { Women } \\
(n=2905)\end{array}$ & $\begin{array}{l}\text { Men } \\
(n=2419)\end{array}$ & $\begin{array}{l}\text { Age } \leq 77^{b} \\
(n=2514)\end{array}$ & $\begin{array}{l}\text { Age }>77 \\
(n=2810)\end{array}$ \\
\hline Cardiac & $219(4.1)$ & $102(3.5)$ & $117(4.8)$ & $85(3.4)$ & $134(4.8)$ \\
\hline Myocardial infarction & $127(2.4)$ & $57(2.0)$ & $70(2.9)$ & $44(1.8)$ & $83(3.0)$ \\
\hline Cardiac arrest & $1(<0.1)$ & $1(<0.1)$ & $0(0.0)$ & $0(0.0)$ & $1(<0.1)$ \\
\hline Pulmonary oedema & $83(1.6)$ & $43(1.5)$ & $40(1.7)$ & $34(1.4)$ & $49(1.7)$ \\
\hline Unstable angina & $8(0.2)$ & $1(<0.1)$ & $7(0.3)$ & $7(0.3)$ & $1(<0.1)$ \\
\hline Vascular & $130(2.4)$ & $83(2.9)$ & $47(1.9)$ & $72(2.9)$ & $58(2.1)$ \\
\hline Pulmonary embolism & $123(2.3)$ & $81(2.8)$ & $42(1.7)$ & $70(2.8)$ & $53(1.9)$ \\
\hline Vessel embolism & $4(0.1)$ & $1(<0.1)$ & $3(0.1)$ & $2(0.1)$ & $2(0.1)$ \\
\hline Aortic dissection & $1(<0.1)$ & $1(<0.1)$ & $0(0.0)$ & $0(0.0)$ & $1(<0.1)$ \\
\hline Aortic rupture & $2(<0.1)$ & $0(0.0)$ & $2(0.1)$ & $0(0.0)$ & $2(0.1)$ \\
\hline Infection and inflammation & $137(2.6)$ & $64(2.2)$ & $73(3.0)$ & $56(2.2)$ & $81(2.9)$ \\
\hline Sepsis & $111(2.1)$ & $50(1.7)$ & $61(2.5)$ & $43(1.7)$ & $68(2.4)$ \\
\hline Epiglottitis & $1(<0.1)$ & $0(0.0)$ & $1(<0.1)$ & $1(<0.1)$ & $0(0.0)$ \\
\hline $\operatorname{SIRS}^{\mathrm{C}}$ & $17(0.3)$ & $11(0.4)$ & $6(0.2)$ & $8(0.3)$ & $9(0.3)$ \\
\hline Other infection $^{d}$ & $8(0.2)$ & $3(0.1)$ & $5(0.2)$ & $4(0.2)$ & $4(0.1)$ \\
\hline \multicolumn{6}{|l|}{ Respiratory } \\
\hline Acute respiratory insufficiency & $78(1.5)$ & $47(1.6)$ & $31(1.3)$ & $47(1.9)$ & $31(1.1)$ \\
\hline Neurological & $14(0.3)$ & $7(0.2)$ & $7(0.3)$ & $3(0.1)$ & $11(0.4)$ \\
\hline Status epilepsia & $1(<0.1)$ & $0(0.0)$ & $1(<0.1)$ & $1(<0.1)$ & $0(0.0)$ \\
\hline Stroke & $11(0.2)$ & $5(0.2)$ & $6(0.2)$ & $2(0.1)$ & $9(0.3)$ \\
\hline $\mathrm{TIA}$ & $2(<0.1)$ & $2(0.1)$ & $0(0.0)$ & $0(0.0)$ & $2(0.1)$ \\
\hline Other $^{\mathrm{e}}$ & $20(0.4)$ & $7(0.2)$ & $13(0.5)$ & $18(0.7)$ & $2(0.1)$ \\
\hline
\end{tabular}

Number (percent);

${ }^{a}$ Patients with multiple occasions included; number missing diagnosis $n=1030$

${ }^{b}$ Median age in years

c Systemic inflammatory response syndrome (SIRS) of non-infectious origin with acute dysfunction

d Pneumonitis, pericarditis, perotonitis, hemorrhagic fever

e Various reasons: acidosis, acute kidney failures, acute intoxications 
Table 2 Age- adjusted relationships between time-sensitive diagnosis and sex, previous history, time intervals, and clinical observations on arrival of PENs, as well as age itself, among unique patients with a final diagnosis

\begin{tabular}{|c|c|c|c|c|c|}
\hline & \multirow{3}{*}{$\begin{array}{l}\text { All } \\
\text { patients } \\
(n= \\
3794)^{a}\end{array}$} & \multicolumn{2}{|c|}{ Time-sensitive diagnosis } & & \\
\hline & & \multirow{2}{*}{$\begin{array}{l}\text { Yes } \\
(n=487)\end{array}$} & \multirow{2}{*}{$\begin{array}{l}\text { No } \\
(n=3307)\end{array}$} & \multicolumn{2}{|c|}{ Age adjusted } \\
\hline & & & & p & OR $(95 \% \mathrm{Cl})$ \\
\hline Age & $79(53,91)$ & $80(61,91)$ & $79(52,91)$ & 0.02 & \\
\hline$<=65$ & 753 (19.8) & $72(14.8)$ & $681(20.6)$ & & 1 \\
\hline $66-80$ & $1327(35.0)$ & $183(37.6)$ & $1144(34.6)$ & & $1.51(1.13,2.02)$ \\
\hline$>80$ & $1714(45.2)$ & $232(47.6)$ & $1482(44.8)$ & & $1.48(1.12,1.96)$ \\
\hline Male sex & $1746(46.0)$ & $239(49.1)$ & 1507 (45.6) & 0.11 & $1.17(0.96,1.41)$ \\
\hline Dyspnoea $(319)^{*}$ & $2867(82.5)$ & $324(76.2)$ & $2543(83.4)$ & $<0.0001$ & $0.58(0.46,0.75)$ \\
\hline Ischemic heart disease (21) & $1017(27.0)$ & $149(30.7)$ & $868(26.4)$ & 0.16 & $1.17(0.94,1.44)$ \\
\hline Heart failure (19) & $1163(30.8)$ & $159(32.7)$ & $1004(30.5)$ & 0.78 & $1.03(0.84,1.27)$ \\
\hline Hypertension & $1905(50.2)$ & $280(57.5)$ & $1625(49.1)$ & 0.007 & $1.32(1.08,1.61)$ \\
\hline Diabetes & $796(21.0)$ & $130(26.7)$ & $666(20.1)$ & 0.002 & $1.41(1.13,1.76)$ \\
\hline Atrial fibrillation (1) & $1168(30.8)$ & $116(23.8)$ & 1052 (31.8) & $<0.0001$ & $0.59(0.47,0.75)$ \\
\hline Pulmonary disease (72) & $1537(41.3)$ & $127(26.5)$ & $1410(43.5)$ & $<0.0001$ & $0.45(0.36,0.56)$ \\
\hline Renal disease (1) & $465(12.3)$ & $79(16.2)$ & $386(11.7)$ & 0.01 & $1.40(1.08,1.83)$ \\
\hline System disease (4) & $270(7.1)$ & $30(6.2)$ & $240(7.3)$ & 0.32 & $0.82(0.55,1.22)$ \\
\hline Cancer (8) & $767(20.3)$ & $88(18.1)$ & $679(20.6)$ & 0.09 & $0.80(0.63,1.03)$ \\
\hline Psychiatric disorder (11) & $615(16.3)$ & $62(12.8)$ & $553(16.8)$ & 0.06 & $0.76(0.58,1.02)$ \\
\hline Pain (178) & $913(25.2)$ & $127(28.4)$ & $786(24.8)$ & 0.06 & $1.24(0.99,1.55)$ \\
\hline Syncope (18) & $53(1.4)$ & $15(3.1)$ & $38(1.2)$ & 0.0005 & $2.95(1.60,5.42)$ \\
\hline Respiratory rate $<8$ or $>25$ (breaths/min)(81) & $2020(54.4)$ & $345(72.3)$ & $1675(51.8)$ & $<0.0001$ & $2.38(1.92,2.95)$ \\
\hline Oxygen saturation < 90 (\%) (74) & $1446(38.9)$ & $282(59.5)$ & $1164(35.9)$ & $<0.0001$ & $2.56(2.10,3.13)$ \\
\hline Systolic blood pressure < $90(\mathrm{mmHg})(133)$ & $84(2.3)$ & $25(5.4)$ & $59(1.8)$ & $<0.0001$ & $3.03(1.87,4.89)$ \\
\hline Heart rate $<40$ or $>120$ (beats/min)(80) & $488(13.1)$ & $116(24.6)$ & $372(11.5)$ & $<0.0001$ & $2.59(2.04,3.28)$ \\
\hline Body temperature $<35.0$ or $>41.0\left({ }^{\circ} \mathrm{C}\right)(129)$ & $23(0.6)$ & $8(1.8)$ & $15(0.5)$ & 0.002 & $3.95(1.66,9.42)$ \\
\hline Degree of consciousness (RLS) > 2 (694) & $67(2.2)$ & $31(8.1)$ & $36(1.3)$ & $<0.0001$ & $6.55(3.99,10.74)$ \\
\hline Pathological ECG (284) & $1110(31.6)$ & $243(52.6)$ & $867(28.4)$ & $<0.0001$ & $2.76(2.26,3.37)$ \\
\hline Time from symptom onset to call (193) & $58(1340)$ & $27(0,223)$ & $62(1341)$ & $<0.0001$ & \\
\hline$<=12 \mathrm{~h}$ & $1076(29.9)$ & $185(41.1)$ & $891(28.3)$ & & 1 \\
\hline $12-24 h$ & $179(5.0)$ & $22(4.9)$ & $157(5.0)$ & & $0.66(0.41,1.06)$ \\
\hline $24-48 \mathrm{~h}$ & $320(8.9)$ & $36(8.0)$ & $284(9.0)$ & & $0.60(0.41,0.88)$ \\
\hline $48-72 \mathrm{~h}$ & $391(10.9)$ & $54(12.0)$ & $337(10.7)$ & & $0.75(0.54,1.04)$ \\
\hline$>72 \mathrm{~h}$ & $1635(45.4)$ & $153(34.0)$ & $1482(47.0)$ & & $0.49(0.39,0.61)$ \\
\hline Time from call to PENs arrival (51) & $18(9,51)$ & $15(8,43)$ & $18(9.51)$ & $<0.0001$ & \\
\hline $0-6 \min$ & $90(2.4)$ & $18(3.8)$ & $72(2.2)$ & & 1 \\
\hline $7-12 \min$ & $929(24.8)$ & $155(32.5)$ & $774(23.7)$ & & $0.79(0.46,1.36)$ \\
\hline $13-24 \min$ & 1527 (40.8) & 176 (36.9) & $1351(41.4)$ & & $0.51(0.30,0.88)$ \\
\hline$>24 \min$ & 1197 (32.0) & $128(26.8)$ & 1069 (32.7) & & $0.47(0.27,0.81)$ \\
\hline
\end{tabular}

Median (10th,90th percentile) or number (percent); ${ }^{*}$ number missing

a Unique patients, randomly selected occasions included 
The following were associated with a decreased risk: a history of atrial fibrillation and pulmonary disease and an increasing delay from symptom onset until the call for EMS (Table 3).

\section{Age-adjusted relationships between 30- day mortality and sex, previous history, time intervals, and clinical observations on the arrival of PENs among unique patients}

Approximately $11 \%$ of all unique patients died within 30 days. Among them, the most common deviations from normal were an abnormal respiratory rate (67.9\%), a low oxygen saturation (60.3\%), and a pathological ECG (38.1\%).

The following were associated with an increased risk of death: increasing age, male sex, a history of renal disease and cancer, deviation from normal in any of the six VS, and a pathologic ECG. Only a history of pulmonary disease was associated with a decreased risk (Table 4).

Multivariable analysis of factors independently associated with 30-day mortality using multiple imputations (4587 patients, $\mathbf{5 0 0}$ died within $\mathbf{3 0}$ days)

The following were significantly independently associated with an increased risk of death: increasing age, a history of renal disease and cancer, an abnormal respiratory rate, a low oxygen saturation, a low systolic blood pressure, a decreased level of consciousness, and an

Table 3 Multivariable analysis of factors independently associated with a time-sensitive diagnosis using multiple imputations (3794 patients, 487 with a time-sensitive diagnosis)

\begin{tabular}{|c|c|c|}
\hline & \multicolumn{2}{|c|}{ Multiple imputations } \\
\hline & $\mathrm{OR}^{\mathrm{a}}(95 \% \mathrm{Cl})^{\mathrm{a}}$ & $p$ \\
\hline Hypertension & $1.45(1.17,1.79)$ & 0.0006 \\
\hline Atrial fibrillation & $0.55(0.43,0.70)$ & $<0.0001$ \\
\hline Pulmonary disease & $0.42(0.33,0.53)$ & $<0.0001$ \\
\hline Renal disease & $1.53(1.15,2.05)$ & 0.004 \\
\hline Pain & $1.42(1.12,1.81)$ & 0.004 \\
\hline Respiratory rate $<8$ or $>25$ (breaths/min) & $1.75(1.37,2.22)$ & $<0.0001$ \\
\hline Saturation < $90(\%)$ & $2.02(1.61,2.53)$ & $<0.0001$ \\
\hline Heart rate $<40$ or $>120$ (rate/min) & $2.08(1.60,2.70)$ & $<0.0001$ \\
\hline Degree of consciousness (RLS) $>2$ & $4.83(2.77,8.44)$ & $<0.0001$ \\
\hline Pathological ECG & $2.46(1.99,3.03)$ & $<0.0001$ \\
\hline Time from symptom to call & & 0.004 \\
\hline$<=12 \mathrm{~h}$ & 1 & \\
\hline $12-24 h$ & $0.69(0.41,1.15)$ & \\
\hline $24-48 h$ & $0.62(0.41,0.93)$ & \\
\hline $48-72 \mathrm{~h}$ & $0.86(0.61,1.22)$ & \\
\hline$>72 \mathrm{~h}$ & $0.64(0.50,0.82)$ & \\
\hline
\end{tabular}

a odds ratio with corresponding $95 \%$ confidence interval abnormal body temperature. Only a history of pulmonary disease was associated with a decreased risk (Table 5).

\section{Discussion}

Among all EMS-assigned patients that were classified as having dyspnoea as the main symptom by the PENs, $11.5 \%$ had a time-sensitive diagnosis, which means that the same patient could have had a time-sensitive diagnosis more than once. Among all EMS-assigned unique patients, approximately $13 \%$ had time-sensitive final diagnoses and among them approximately $27 \%$ died within 30 days.

The most important information was that aspects of the patients previous history and deviation from normal in several vital parameters were independently associated with both a time-sensitive final diagnosis as well the risk of death within 30 days. But symptoms of pain, a pathologic ECG, and a short delay from onset of symptoms until the call for EMS were significantly associated only with a time-sensitive diagnosis, whereas increasing age was associated only with the risk of death but not a time-sensitive diagnosis.

The finding that increasing age was significantly associated only with death but not with a time-sensitive diagnosis indicates that different mechanisms operate in attaining either of these endpoints. It may be that some of the time-sensitive diagnoses are particularly dangerous among the elderly. It may also be that some other diseases, which are not defined as time-sensitive diagnoses may still be life-threatening among the elderly. Previous studies $[12,13]$ emphasize that increasing age and especially older elderly ( $>80$ years) are more vulnerable, in addition to experiencing severe conditions. They are even more fragile due to their reduced capacity in managing their care and suffer from a decline in physiological reserves. Also, their physiological coping strategies in managing severe conditions may function differently, depending on how affected they are by their comorbidity.

A history of either hypertension or renal disease were significantly associated with a time-sensitive diagnosis. In the general population, the risk of hypertension increases with age $[14,15]$ and uncontrolled hypertension is a powerful and independent risk factor for cardiovascular morbidity and mortality as well as all-cause death. Hypertension is known as the silent killer because it has no specific symptoms and no early warning signs [14, 16-18].

The renal function decreases with increasing age and the prevalence of chronic kidney disease (CKD) increases steadily among people aged $>65$ years [19-21].This is associated with an increased risk of death as well as cardiovascular disease and progression to renal failure [21, 22]. Sustained hypertension may lead to declines in kidney function and 
Table 4 Age- adjusted relationships between 30- day mortality and sex, previous history, time intervals, and clinical observations on arrival of PENs among unique patients

\begin{tabular}{|c|c|c|c|c|c|}
\hline & \multirow{3}{*}{$\begin{array}{l}\text { All } \\
\text { patients } \\
(n= \\
4587)^{a}\end{array}$} & \multicolumn{2}{|c|}{ Dead within 30 days } & \multirow{2}{*}{\multicolumn{2}{|c|}{ Age adjusted }} \\
\hline & & \multirow{2}{*}{$\begin{array}{l}\text { Yes } \\
(n=500)\end{array}$} & \multirow{2}{*}{$\begin{array}{l}\text { No } \\
(n= \\
4087)\end{array}$} & & \\
\hline & & & & $p$ & OR $(95 \% \mathrm{Cl})$ \\
\hline Age & $77(43,91)$ & $83(68,93)$ & $76(39,90)$ & $<0.0001$ & \\
\hline$<=65$ & $1197(26.1)$ & $38(7.6)$ & $1159(28.4)$ & & 1 \\
\hline $66-80$ & 1505 (32.8) & $170(34.0)$ & $1335(32.7)$ & & $3.88(2.71,5.57)$ \\
\hline$>80$ & $1885(41.1)$ & $292(58.4)$ & $1593(39.0)$ & & $5.59(3.95,7.90)$ \\
\hline Male sex & $2051(44.7)$ & $252(50.4)$ & $1799(44.0)$ & 0.002 & $1.36(1.12,1.64)$ \\
\hline Dyspnoea $(418)^{*}$ & $3302(79.2)$ & $371(83.4)$ & $2931(78.7)$ & 0.96 & $0.99(0.76,1.30)$ \\
\hline Ischemic heart disease (21) & $1140(25.0)$ & $131(26.3)$ & $1009(24.8)$ & 0.07 & $0.82(0.66,1.02)$ \\
\hline Heart failure (31) & $1262(27.7)$ & $182(36.7)$ & $1080(26.6)$ & 0.16 & $1.16(0.94,1.41)$ \\
\hline Hypertension (8) & $2114(46.2)$ & $256(51.2)$ & $1858(45.6)$ & 0.15 & $0.87(0.71,1.05)$ \\
\hline Diabetes (12) & $878(19.2)$ & $106(21.2)$ & $772(18.9)$ & 0.76 & $1.04(0.82,1.31)$ \\
\hline Atrial fibrillation (13) & $1277(27.9)$ & $175(35.1)$ & $1102(27.0)$ & 0.92 & $1.01(0.82,1.24)$ \\
\hline Pulmonary disease (89) & 1789 (39.8) & $169(34.2)$ & $1620(40.5)$ & 0.004 & $0.74(0.61,0.91)$ \\
\hline Renal disease (12) & 499 (10.9) & $83(16.6)$ & $416(10.2)$ & 0.006 & $1.44(1.11,1.87)$ \\
\hline System disease (15) & $307(6.7)$ & $40(8.0)$ & $267(6.6)$ & 0.55 & $1.11(0.78,1.58)$ \\
\hline Cancer (17) & $863(18.9)$ & $149(29.9)$ & $714(17.5)$ & $<0.0001$ & $1.67(1.35,2.07)$ \\
\hline Psychiatric disorder (30) & $828(18.2)$ & $74(14.8)$ & 754 (18.6) & 0.84 & $0.97(0.75,1.27)$ \\
\hline Pain (206) & $1098(25.1)$ & 89 (19.6) & $1009(25.7)$ & 0.05 & $0.78(0.61,0.99)$ \\
\hline Syncope (19) & $63(1.4)$ & $9(1.8)$ & $54(1.3)$ & 0.20 & $1.62(0.78,3.36)$ \\
\hline Respiratory rate $<8$ or $>25$ (breaths/min)(105) & $2104(46.9)$ & $332(67.9)$ & $1772(44.4)$ & $<0.0001$ & $2.19(1.78,2.68)$ \\
\hline Oxygen saturation < 90 (\%) (90) & $1485(33.0)$ & $296(60.3)$ & $1189(29.7)$ & $<0.0001$ & $3.06(2.52,3.73)$ \\
\hline Systolic blood pressure < 90 (mmHg) (178) & $90(2.0)$ & $37(7.8)$ & $53(1.3)$ & $<0.0001$ & $6.45(4.12,10.10)$ \\
\hline Heart rate $<40$ or $>120$ (beats/min) (95) & $517(11.5)$ & $84(17.1)$ & $433(10.8)$ & $<0.0001$ & $1.80(1.39,2.34)$ \\
\hline Body temperature $<35.0$ or $>41.0\left({ }^{\circ} \mathrm{C}\right)(187)$ & $24(0.5)$ & $10(2.1)$ & $14(0.4)$ & $<0.0001$ & $6.38(2.70,15.04)$ \\
\hline Degree of consciousness (RLS) > 2 (816) & $70(1.9)$ & $27(7.2)$ & $43(1.3)$ & $<0.0001$ & $5.56(3.35,9.23)$ \\
\hline Pathological ECG (690) & $1153(29.6)$ & $173(38.1)$ & $980(28.5)$ & 0.002 & $1.38(1.13,1.70)$ \\
\hline Time from symptom to call (332) & $51(0,339)$ & $50(0,318)$ & $51(0,340)$ & 0.41 & \\
\hline$<=12 \mathrm{~h}$ & $1470(34.5)$ & $163(34.8)$ & $1307(34.5)$ & & 1 \\
\hline $12-24 h$ & $210(4.9)$ & $20(4.3)$ & $190(5.0)$ & & $0.79(0.48,1.30)$ \\
\hline $24-48 \mathrm{~h}$ & $357(8.4)$ & $37(7.9)$ & $320(8.5)$ & & $0.86(0.59,1.26)$ \\
\hline $48-72 \mathrm{~h}$ & $420(9.9)$ & $50(10.7)$ & $370(9.8)$ & & $0.94(0.67,1.33)$ \\
\hline$>72 \mathrm{~h}$ & $1798(42.3)$ & $199(42.4)$ & $1599(42.2)$ & & $0.89(0.71,1.12)$ \\
\hline Time from call to EMS arrival (58) & $18(9,50)$ & $16(8,44)$ & $18(9,51)$ & 0.02 & \\
\hline $0-6 \min$ & $100(2.2)$ & $16(3.2)$ & $84(2.1)$ & & 1 \\
\hline $7-12 \min$ & $1110(24.5)$ & $135(27.2)$ & $975(24.2)$ & & $0.69(0.39,1.23)$ \\
\hline $13-24 \min$ & $1853(40.9)$ & $198(39.8)$ & $1655(41.0)$ & & $0.59(0.33,1.04)$ \\
\hline$>24 \min$ & $1466(32.4)$ & $148(29.8)$ & $1318(32.7)$ & & $0.55(0.31,0.98)$ \\
\hline
\end{tabular}

median (10th,90th percentile) or number (percent); * number missing

${ }^{a}$ Unique patients, randomly selected occasions included 
Table 5 Multivariable analysis of factors independently associated with 30-day mortality using multiple imputations (4587 patients, 500 died within 30 days)

\begin{tabular}{lll}
\hline & Multiple imputations $\mathbf{( 5 0 0 + 4 0 8 7 )}$ & $\mathbf{p}$ \\
\cline { 2 - 3 } & $\mathbf{O R}^{\mathbf{a}} \mathbf{( 9 5 \% \mathbf { C l } ) ^ { \mathbf { a } }}$ & $<0.0001$ \\
\hline Age & 1 & 1 \\
$\quad<=65$ & $2.57(1.76,3.75)$ & 0.0004 \\
$66-80$ & $3.68(2.56,5.28)$ & 0.007 \\
$\quad>80$ & $0.68(0.55,0.84)$ & $<0.0001$ \\
Pulmonary disease & $1.46(1.11,1.91)$ & 0.0003 \\
Renal disease & $1.81(1.45,2.25)$ & $<0.0001$ \\
Cancer & $1.52(1.21,1.90)$ & $<0.0001$ \\
Respiratory rate $<8$ or $>25$ (breaths/min) & $2.61(2.10,3.24)$ & 0.001 \\
Saturation $<90$ (\%) & $5.13(3.18,8.27)$ & $<0.0001$ \\
Systolic blood pressure $<90(\mathrm{mmHg})$ & $4.41(1.78,10.96)$ & \\
Body temperature $<35.0$ or $>41.0\left({ }^{\circ} \mathrm{C}\right)$ & $3.61(2.08,6.29)$ & \\
Degree of consciousness $(\mathrm{RLS})>2$ & & \\
\hline
\end{tabular}

a Odds ratio with corresponding confidence interval

progressive failure in kidney function can conversely lead to worsening blood pressure control. As a consequence, it can lead to fluid accumulation inside the body with dyspnoea as a symptom that may persist despite optimal care in these patients [23-28]. Hypertension in combination with CKD poses a high risk for adverse outcomes depending on the severity level of renal failure [29-31].

A low systolic blood pressure predicted death within 30 days. Similar findings have been seen in EMS patients with chest pain, where a low systolic blood pressure was a strong predictor of acute- life-threatening conditions [32], as well as a predictor of death within 30 days [33]. A low systolic blood pressure is a hallmark of critical illness [34, 35 ] and a predictor of poor outcome in conditions such as acute myocardial infarction, sepsis or pulmonary embolism (where dyspnoea may be a presenting symptom) [34]. The assessment of the severity of pulmonary embolism is particularly based on the presence of hypotension or signs of shock and respiratory failure [36-38].

An abnormal body temperature $\left(<35^{\circ} \mathrm{C}\right.$ or $\left.>41^{\circ} \mathrm{C}\right)$ was significantly associated with death within 30 days. One reason may be physiological changes among the elderly, which tend to reduce the ability of organ systems to adapt to pathological changes. Thus, body temperature tends to be lower and the ability of the body to change with different stressors is reduced. Therefore, fever in an older patient often indicates a more severe infection and is associated with life-threatening consequences $[15,39]$.

A decreased level of consciousness was significantly associated with both death and a time-sensitive diagnosis. In unselected acutely and/or critically ill patients admitted to ED [35, 40], decreased level of consciousness was the most important predictor of early death. In EMS patients diagnosed with respiratory diseases in hospitals
[6], the 30- day mortality was highest if decreased level of consciousness.

Reasons for decreased level of consciousness among patients with dyspnoea could be related to prolonged hypoxia and/or increased levels of carbon dioxide $\left(\mathrm{CO}_{2}\right)$ $[41,42]$. An abnormal respiratory rate is a more sensitive marker than other VS in identifying critically ill patients, as the body attempts to correct hypoxaemia and hypercarbia, by increasing respiratory rate. An abnormally low respiratory rate is often associated with a reduced level of consciousness [43].

A low oxygen saturation was associated with adverse outcomes in agreement with findings in medical patients in EDs [35, 44]. Previous results [35] claim that pulse oximetry lacks specificity and is therefore not a specific indicator for serious illness, since it does not mirror the quality of respiration and ventilation as it does not give information about the carbon dioxide $\left(\mathrm{CO}_{2}\right)$ status.

Experiences from critically ill patients [40] indicate that the number of abnormal VS is associated with the risk of death. However, in patients with sepsis [45], VS may only be vague in prediction of the disease and can be normal. Instead, other symptoms and signs are common, (e.g. pain, acute altered mental status, leg weakness, and dyspnoea). In our study, only $39 \%$ had a pulmonary cause of the underlying infection, which indicated that dyspnoea is frequent in sepsis. This could indicate that the presence of dyspnoea is a part of a systemic pathophysiological response to the underlying infection, which may include an anaerobic metabolism and metabolic acidosis.

ECG pathology may be one of the most important signs predicting adverse outcome [32] and therefore a cornerstone in early detection of a time-sensitive 
diagnosis such as myocardial infarction [46]. ECG also have an important role in detecting severe arrhythmias, which can be the cause of dyspnoea and may lead to a time-sensitive diagnosis if not noticed [47]. A large proportion had a history of atrial fibrillation [8], which indicated that it was not a new finding that caused adverse illness among these patients.

The fact that pain was associated with a time-sensitive diagnosis is not unexpected, as this is previously described $[38,48,49]$. Pain may be due to the loss of perfusion (angina, myocardial infarction) and low oxygen tension (hypoxia) with resulting inflammatory response [50].

\section{Strengths and limitations}

A strength is the large sample size, and the fact that all records were manually reviewed. The study is limited to southwest part of Sweden, which could hamper generalisability to other settings. Thus, in the north of Sweden there are longer transport distances which may lead to fluctuations. Furthermore, data were retrospectively collected from patient records and VS and other clinical parameters could have been measured but never reported. There was sometimes insufficient documentation. Dyspnoea could be present in other medical conditions such as chest pain. Thus, some patients with dyspnoea may have been classified to other ESS codes (not ESS 04).

The final diagnosis was missing in 1030 cases explained by patients left on-scene and patients transported to the ED who were directly sent home. However, with the intention to reduce bias associated with medical record reviews, the study was conducted in agreement with the paper by Kaji et al. [51].

\section{Conclusion}

Among patients with dyspnoea as the main symptom, several factors including age, previous medical history, deviating VS, the ECG pattern, symptoms of pain and the delay between onset of symptoms and the call for EMS are important to consider in the early risk assessment. The development of a decision support tool may increase the possibility to differentiate patients with time-sensitive conditions, from those without, at an early stage.

\section{Supplementary Information}

Supplementary information accompanies this paper at https://doi.org/10. 1186/s12873-020-00384-1.

Additional file 1. Deviating vital signs Red/Orange level according to RETTS-A (2017 version)

\section{Abbreviations}

CKD: Chronic kidney disease; ECG: Electrocardiogram; ED: Emergency department; EMS: Emergency medical services; ESS: Emergency Symptoms and Signs; ICD-10: International Statistical Classification of Diseases and Related Health Problems - 10th revision; PEN: Pre-hospital emergency nurse; RETTS-A: Rapid Emergency Triage and Treatment System for adults; VS: Vital signs

\section{Acknowledgements}

The authors wish to acknowledge the help providing by Jonny Lindqvist, Region Västra Götaland, Gothenburg, Sweden in creating the data protocol. We also acknowledge the EMS organisations in both Södra Älvsborg hospital in Borås and Sahlgrenska University Hospital in Gothenburg, Sweden for providing data on EMS missions.

\section{Ethical approval and consent to participate}

The study was approved by The Regional Ethical Review Board in Gothenburg, Sweden (Dnr 989-17). All procedures that were performed were in agreement with the ethical standards of the Declaration of Helsinki [52]. To use the data for analysis, consents were obtained from chief executive officers in the two EMS organisations. In agreement with the Swedish law there is often a requirement for informed consent for participation, but in this study informed consent was not required for participation. Ethical Review Boards in Sweden declare that informed consent does not apply if it involves a disproportionately large work effort. Patients integrity remained intact as their identification number was translated to a unique code.

Also, some patients could never give informed consent retrospectively since they were either in a very poor condition or had died. The risk of selection bias would thereby increase.

\section{Authors' contributions}

WK, CA, JH, and LP developed the design and the research protocol. WK, CA, and $\mathrm{JH}$ created the data forms. WK collected the data. TK and CM supervised the statistical analyses and discussed with WK, CA and JH. WK wrote the first draft of the manuscript. WK, CA, JH, LP, TK and CM supervised and analysed the data and their interpretation. All the authors participated in the final drafting of the manuscript and have read and approved the final manuscript

\section{Funding}

WK received funding from the University of Borås, Sweden. The funder did not have any role in the study design, aim, or process. Open Access funding provided by University of Boras.

\section{Availability of data and materials}

The datasets analysed during the current study are available from the corresponding author on reasonable request.

Consent for publication

Not applicable.

\section{Competing interests}

The authors declare that they have no competing interests.

\section{Author details}

${ }^{1}$ PreHospen- Centre for Pre-hospital Research, Faculty of Caring, Work Life and Social Welfare, University of Borås, SE-501 90 Borås, Sweden. ${ }^{2}$ Faculty of Caring Science, Work Life and Social Welfare, University of Borås, Borås, Sweden. ${ }^{3}$ Biostatistics, School of Public Health and Community Medicine, Institute of Medicine, Sahlgrenska Academy, University of Gothenburg, Gothenburg, Sweden. ${ }^{4}$ Department of Molecular and Clinical Medicine, Sahlgrenska Academy, University of Gothenburg, Gothenburg, Sweden.

Received: 14 September 2020 Accepted: 30 October 2020

Published online: 10 November 2020

References.

1. Coccia CB, Palkowski GH, Schweitzer B, Motsohi T, Ntusi NA. Dyspnoea. Pathophysiology and a clinical approach. S Afr Med J. 2016;106:32-6.

2. Lansing RW, Gracely RH, Banzett RB. The multiple dimensions of dyspnea: review and hypotheses. Respir Physiol Neurobiol. 2009;167:53-60.

3. Wibring K, Magnusson C, Axelsson C, Lundgren P, Herlitz J, Andersson HM. Towards definitions of time-sensitive conditions in prehospital care. Scand Trauma Resusc Emerg Med. 2020;28:7. 
4. Carel H, Macnaughton J, Dodd J. Invisible suffering: breathlessness in and beyond the clinic. Lancet Respir Med. 2015;3:278-9.

5. Kelly AM, Holdgate A, Keijzers G, Klim S, Graham CA, Craig S, et al. Epidemiology, prehospital care and outcomes of patients arriving by ambulance with dyspnoea: an observational study. Scand J Trauma Resusc Emerg Med. 2016;24:113.

6. Lindskou TA, Pilgaard L, Sovso MB, Klojgard TA, Larsen TM, Jensen FB, et al. Symptom, diagnosis and mortality among respiratory emergency medical service patients. PLoS One. 2019;14:e0213145.

7. Prekker ME, Feemster LC, Hough CL, Carlbom D, Crothers K, Au DH, et al. The epidemiology and outcome of prehospital respiratory distress. Acad Emerg Med. 2014;21:543-50.

8. Kauppi W, Herlitz J, Magnusson C, Palmer L, Axelsson C. Characteristics and outcomes of patients with dyspnoea as the main symptom, assessed by prehospital emergency nurses- a retrospective observational study. BMC Emerg Med. 2020;20:67.

9. Suserud BO. A new profession in the pre-hospital care field--the ambulance nurse. Nurs Crit Care. 2005;10:269-71.

10. Widgren BR, Jourak M. Medical emergency triage and treatment system (METTS): a new protocol in primary triage and secondary priority decision in emergency medicine. J Emerg Med. 2011;40:623-8.

11. Sovso MB, Christensen MB, Bech BH, Christensen HC, Christensen EF, Huibers L. Contacting out-of-hours primary care or emergency medical services for time-critical conditions - impact on patient outcomes. BMC Health Serv Res. 2019;19:813.

12. Hegendorfer E, Vaes B, Mathei C, Van Pottelbergh G, Degryse JM. Correlates of dyspnoea and its association with adverse outcomes in a cohort of adults aged 80 and over. Age Ageing. 2017;46:994-1000.

13. Landi F, Calvani R, Tosato M, Martone AM, Bernabei R, Onder G, et al. Impact of physical function impairment and multimorbidity on mortality among community-living older persons with sarcopaenia: results from the iSIRENTE prospective cohort study. BMJ Open. 2016;6:e008281.

14. World HO. A global brief on hypertension : silent killer, global public health crisis: World health day 2013. Geneva: World Health Organization; 2013.

15. Chester JG, Rudolph JL. Vital signs in older patients: age-related changes. J Am Med Dir Assoc. 2011;12:337-43.

16. Mensah GA. Commentary: hypertension phenotypes: the many faces of a silent killer. Ethn Dis. 2019;29:545-8.

17. Lim SS, Vos T, Flaxman AD, Danaei G, Shibuya K, Adair-Rohani H, et al. A comparative risk assessment of burden of disease and injury attributable to 67 risk factors and risk factor clusters in 21 regions, 1990-2010: a systematic analysis for the global burden of disease study 2010. Lancet. 2012;380:2224-60.

18. Janjua G, Guldenring D, Finlay D, McLaughlin J. Wireless chest wearable vital sign monitoring platform for hypertension. Conf Proc IEEE Eng Med Biol Soc. 2017;2017:821-4.

19. Iseki K. Factors influencing the development of end-stage renal disease. Clin Exp Nephrol. 2005;9:5-14.

20. Iseki K. Renal outcomes in chronic kidney disease. Nephrology (Carlton). 2010;2:27-30.

21. Saran R, Robinson B, Abbott KC, Bragg-Gresham J, Chen X, Gipson D, et al. US renal data system 2019 annual data report: epidemiology of kidney disease in the United States. Am J Kidney Dis. 2020;75:A6-7.

22. Moody WE, Edwards NC, Chue CD, Ferro CJ, Townend JN. Arterial disease in chronic kidney disease. Heart. 2013;99:365-72.

23. Judd E, Calhoun DA. Management of hypertension in CKD: beyond the guidelines. Adv Chronic Kidney Dis. 2015;22:116-22.

24. Udani S, Lazich I, Bakris GL. Epidemiology of hypertensive kidney disease. Nat Rev Nephrol. 2011;7:11-21.

25. Kazancioglu R. Risk factors for chronic kidney disease: an update. Kidney Int Suppl (2011). 2013;3:368-71.

26. Ritz E. Hypertension and kidney disease. Clin Nephrol. 2010;74:S39-43.

27. Tedla FM, Brar A, Browne R, Brown C. Hypertension in chronic kidney disease: navigating the evidence. Int J Hypertens. 2011;2011:132405.

28. Salerno FR, Parraga G, Mclntyre CW. Why is your patient still short of breath? Understanding the complex pathophysiology of dyspnea in chronic kidney disease. Semin Dial. 2017;30:50-7.

29. Wen CP, Cheng TY, Tsai MK, Chang YC, Chan HT, Tsai SP, et al. All-cause mortality attributable to chronic kidney disease: a prospective cohort study based on 462293 adults in Taiwan. Lancet. 2008;371:2173-82.

30. Collins AJ, Foley RN, Chavers B, Gilbertson D, Herzog C, Johansen K, et al. United States renal data system 2011 annual data report: atlas of chronic kidney disease \& end-stage renal disease in the United States. Am J Kidney Dis. 2012;59(A7):e1-420.

31. Ridao N, Luno J. Garcia de Vinuesa S, Gomez F, Tejedor a, Valderrabano F. prevalence of hypertension in renal disease. Nephrol Dial Transplant. 2001; 16:70-3.

32. Wibring K, Herlitz J, Christensson L, Lingman M, Bang A. Prehospital factors associated with an acute life-threatening condition in non-traumatic chest pain patients - a systematic review. Int J Cardiol. 2016;219:373-9.

33. Herlitz J, Hansson E, Ringvall E, Starke M, Karlson BW, Waagstein L. Predicting a life-threatening disease and death among ambulancetransported patients with chest pain or other symptoms raising suspicion of an acute coronary syndrome. Am J Emerg Med. 2002;20:588-94.

34. Jones $A E$, Yiannibas V, Johnson C, Kline JA. Emergency department hypotension predicts sudden unexpected in-hospital mortality: a prospective cohort study. Chest. 2006:130:941-6.

35. Hong W, Earnest A, Sultana P, Koh Z, Shahidah N, Ong ME. How accurate are vital signs in predicting clinical outcomes in critically ill emergency department patients. Eur J Emerg Med. 2013;20:27-32.

36. Jaff MR, McMurtry MS, Archer SL, Cushman M, Goldenberg N, Goldhaber SZ, et al. Management of massive and submassive pulmonary embolism, iliofemoral deep vein thrombosis, and chronic thromboembolic pulmonary hypertension: a scientific statement from the American Heart Association. Circulation. 2011;123:1788-830.

37. Burrowes KS, Clark AR, Tawhai MH. Blood flow redistribution and ventilation-perfusion mismatch during embolic pulmonary arterial occlusion. Pulm Circ. 2011;1:365-76.

38. Agnelli G, Becattini C. Acute pulmonary embolism. N Engl J Med. 2010;363: 266-74

39. Blatteis CM. Age-dependent changes in temperature regulation - a mini review. Gerontology. 2012;58:289-95.

40. Barfod C, Lauritzen MM, Danker JK, Soletormos G, Forberg JL, Berlac PA, et al. Abnormal vital signs are strong predictors for intensive care unit admission and in-hospital mortality in adults triaged in the emergency department - a prospective cohort study. Scand J Trauma Resusc Emerg Med. 2012;20:28.

41. Urwin L, Murphy R, Robertson C, Pollok A. A case of extreme hypercapnia: implications for the prehospital and accident and emergency department management of acutely dyspnoeic patients. Emerg Med J. 2004;21:119-20.

42. Singer Fisher $E$, Burns B. Acute decompensated heart failure: new strategies for improving outcomes. Emerg Med Pract. 2017;19:1-24.

43. Cretikos MA, Bellomo R, Hillman K, Chen J, Finfer S, Flabouris A. Respiratory rate: the neglected vital sign. Med J Aust. 2008;188:657-9.

44. Mower WR, Sachs C, Nicklin EL, Safa P, Baraff LJ. Effect of routine emergency department triage pulse oximetry screening on medical management. Chest. 1995;108:1297-302

45. Wallgren UM, Bohm KEM, Kurland L. Presentations of adult septic patients in the prehospital setting as recorded by emergency medical services: a mixed methods analysis. Scand J Trauma Resusc Emerg Med. 2017;25:23.

46. Olson CW, Wagner GS, Terkelsen CJ, Stickney R, Lim T, Pahlm O, et al. Olson method for locating and calculating the extent of transmural ischemic areas at risk of infarction. J Electrocardiol. 2014;47:430-7.

47. Lip GYH, Coca A, Kahan T, Boriani G, Manolis AS, Olsen MH, et al. Hypertension and cardiac arrhythmias: executive summary of a consensus document from the European heart rhythm association (EHRA) and ESC Council on hypertension, endorsed by the Heart Rhythm Society (HRS), Asia-Pacific Heart Rhythm Society (APHRS), and Sociedad Latinoamericana de Estimulacion Cardiaca y Electrofisiologia (SOLEACE). Eur Heart J Cardiovasc Pharmacother. 2017;3:235-50.

48. Olander A, Andersson H, Sundler AJ, Bremer A, Ljungstrom L, Andersson HM. Prehospital characteristics among patients with sepsis: a comparison between patients with or without adverse outcome. BMC Emerg Med. 2019;19:43.

49. Andersson PO, Lawesson SS, Karlsson JE, Nilsson S, Thylen I, SymTime SG. Characteristics of patients with acute myocardial infarction contacting primary healthcare before hospitalisation: a cross-sectional study. BMC Fam Pract. 2018:19:167.

50. Lokmic Z, Musyoka J, Hewitson TD, Darby IA. Hypoxia and hypoxia signaling in tissue repair and fibrosis. Int Rev Cell Mol Biol. 2012;296:139-85.

51. Kaji AH, Schriger D, Green S. Looking through the retrospectoscope: reducing bias in emergency medicine chart review studies. Ann Emerg Med. 2014;64:292-8 
52. World Medical Association. World medical association declaration of Helsinki: ethical principles for medical research involving human subjects. JAMA. 2013;310:2191-4.

\section{Publisher's Note}

Springer Nature remains neutral with regard to jurisdictional claims in published maps and institutional affiliations.

Ready to submit your research? Choose BMC and benefit from:

- fast, convenient online submission

- thorough peer review by experienced researchers in your field

- rapid publication on acceptance

- support for research data, including large and complex data types

- gold Open Access which fosters wider collaboration and increased citations

- maximum visibility for your research: over $100 \mathrm{M}$ website views per year

At BMC, research is always in progress. 